\title{
Disorder and Functionality in Integrated Photonics (Project Report 0925399-Y1)
}

\author{
SHAYAN MOOKHERJEA \\ Department of Electrical and Computer Engineering, University of California, San Diego, MC 0407 La \\ Jolla CA 92093-0407 USA \\ Email:smookher@ucsd.edu
}

The goal of this NSF-funded project (0925399) is to study a new class of photonic circuits and devices fabricated in high-index contrast semiconductor materials in which disorder plays a significant role.

\section{Introduction and Goals}

Integrated photonics can have transformative impact on optical communications, RF photonics, optically-assisted analog-to-digital conversion, frequency comb generation, and the miniaturization of sensor instruments such as spectrometers towards the "lab on a chip" concept. Large-scale photonic circuits are made using fabrication technologies that are similar to those used by the silicon microelectronics industry [1]. It was estimated by IBM that SOI photonics fabricated using $65 \mathrm{~nm}$ CMOS toolset and $45 \mathrm{~nm}$ CMOS toolset (193 nm lasers, with optical proximity correction) implies an ITRS $3 \sigma$ dimensional tolerance of $35 \mathrm{~nm}$ and $16 \mathrm{~nm}$, respectively, implying that (root mean squared) roughness of approximately $10 \mathrm{~nm}$ or $5 \mathrm{~nm}$ can be expected in the two generations. This cannot be ignored, since some silicon photonic components which are used as building blocks of complex circuits have tolerances of only a few nanometers. For example, the resonance frequencies of microring resonators which differ by a few nanometers in the waveguide height can be different by several hundreds of gigahertz, which is beyond the typical tuning range of standard laser diodes. As another example, disorder in the $5 \mathrm{~nm}$ to 10 $\mathrm{nm}$ range in a photonic crystal waveguide can increase the propagation loss to about $50-100 \mathrm{~dB} / \mathrm{cm}$. As a third example, the traveling-wave phase in a centimeter-long waveguide is perturbed by waveguide roughness. The phase imbalance between two arms of a Mach-Zehnder modulator degrades the extinction ratio and impacts applications in RF photonics.

However, in the words of the physicist J. M. Ziman, "Disorder is not mere chaos: it implies defective order" [2]. Our project goals are to (i) obtain scientific insights into designing optical structures which recognize, and potentially even utilize, disorder for functionality, and (ii) make engineering advances in correcting or compensating for defective order when necessary.

The research activities in this project relate to finding ways to understand, withstand and sometimes use disorder for useful behavior when possible. (One example of the latter is a channelized optical power monitor, which is a variant 
of an optical spectrum analyzer with no moving parts.) Somewhat paradoxically, we find that some of the effects of disorder are more predictable and controllable in longer structures than in shorter ones. Diagnostic tools and design algorithms we developed recently are also more reliable in larger ensembles. This has implications for the types of device structures which are chosen as the building blocks of larger circuits.

The inter-related goals and work-plans of this project are shown schematically in Figure 1. Taken together, the research outcomes of this project will help bridge the gap between optical device engineering and condensed matter physics, and advance our understanding of the cooperative behavior of photons in lithographically patterned structures. The devices of interest here are intended for use as reconfigurable optical buffers, switches, spectrometers and wavelength converters. The benefit of such integrated circuits, when realized, is to avoid using expensive high-power lasers, and mis-alignment prone freespace optical assemblies that use bulk optics, or long lengths of optical fiber and instead efficiently use on-chip sources, modulators, and novel device components realized at the microchip scale.

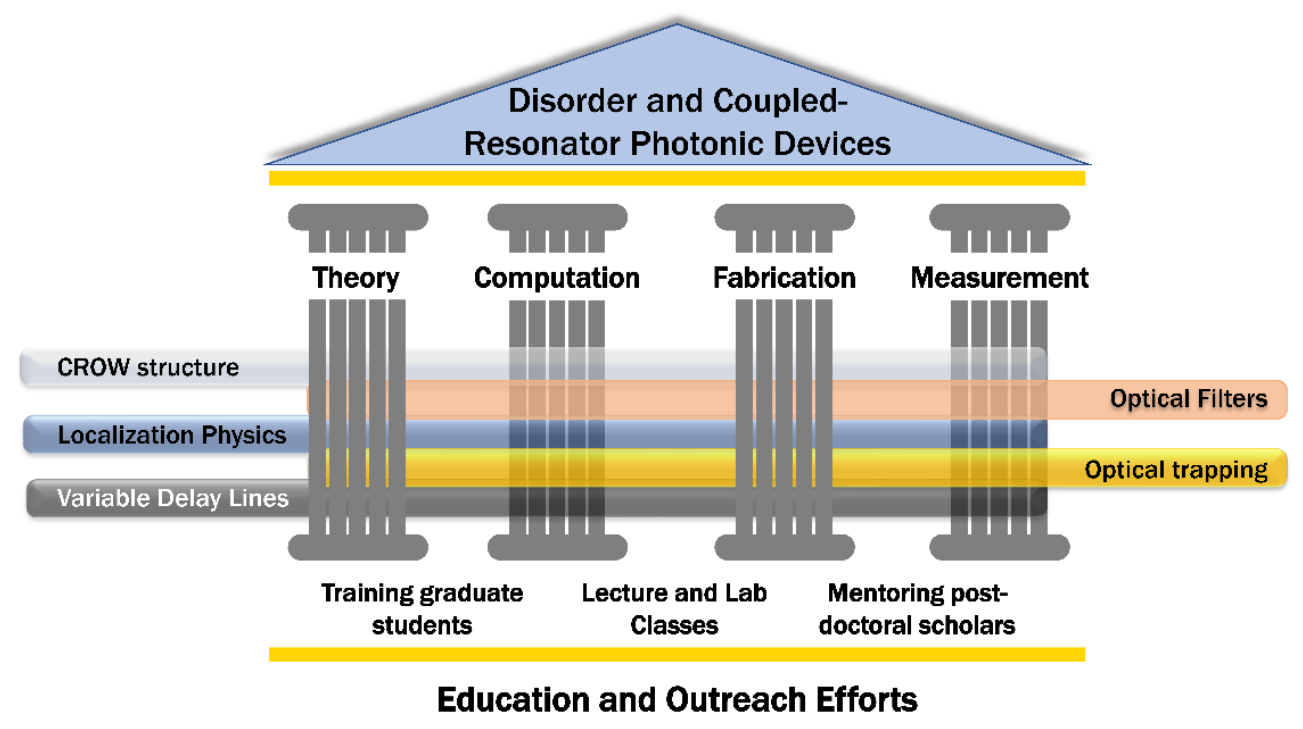

Figure 1 The research goals of this three-year project include five topical areas, each of which intersect four project activities that include research in theory, computation methods, fabrication technologies and measurement. The project also supports educational and outreach efforts as indicated.

\section{Activities}

The principal focus of research during the first year $(08 / 2009-07 / 2010)$ was on developing models to study integrated photonics devices which can comprise several hundred individual elements in periodic structure, such as the 
coupled-resonator optical waveguide (CROW). Potential applications of this structure include efficient nonlinear optics [3]-[5], dispersion compensation, lasers [6] and quantum light generation [7]. In a collaboration with IBM, silicon microring CROWs of up to 235 coupled resonators were successfully fabricated and measured, and a good agreement was achieved between experiments and theory [8]-[10].

The dispersion properties of this structure were studied and compared to earlier theoretical models [11]-[16]. This is an important aspect of this project because while there are simple models of propagation in the tight-binding regime [17] [19], optical propagation near the band-edge regions is difficult to calculate [20]. In this regime, the product of the Bloch K-vector and the localization length (experimentally measured to be about 5 unit cells or $17 \mu \mathrm{m}$ [21]) can be as small as $\mathrm{K}_{\mathrm{B}} \mathrm{l} \sim 0.0475 \mu^{-1} \times 17 \mu \mathrm{m}=0.81$. Since this is less than unity, such structures can be in the strong-localization regime [22], for which simulation methods and computational tools are not as well developed compared to the earlier CROW waveguide models.

Before studying strong localization effects, other aspects of the CROW theory have to be developed in order to successfully fabricate devices. Dispersion (group velocity dispersion, GVD) is not only relevant to the CROW structure as a fundamental principle but is also important in designing directional couplers for ring-based filters. We experimentally studied the coupler dispersion using a microring resonator coupled to a waveguide [23]. An accurate knowledge of dispersion is necessary to design wavelength conversion devices that use four-wave mixing (FWM) and multi-stage devices in which the free-spectral ranges (FSR) of different sections must be precisely related [24].

We developed an extension of coupled-mode theory which is applicable to directional couplers in silicon photonics, coupled-waveguide structures [25] as well as coupled-resonator structures [26]. Based on our analytical coupledresonator models, we developed a model for photon-pair generation using spontaneous four-wave mixing (SFWM) [27]. The third-order optical Kerr nonlinearity which is used as the physical principle for resonator-enhanced FWM and SFWM can also be used in a different way to spatially confine light in a small region, e.g., spatial solitons [28]-[30].

We require an external control "knob" to tune the waveguiding properties of the CROW structure. The nonlinear microring add-drop filter [31] was considered as a building block for a CROW device that was externally tunable (using optical fields). The thermo-optic effect, which we used for tunable filters, was also another option as a control knob [32]. However, as the project progressed, we decided to use electro-optic tunability for most of our studies. Using electro-optic control fields was practically convenient since it was easy to generate the switching waveforms in the laboratory [33]. 


\section{Progress and Impact}

In the first year of this project, we designed coupled-microring filters and coupled microring CROW structures in silicon photonics. The longest structures consist of up to 235 coupled microring resonators and use about 470 directional couplers. Careful attention was paid to the design and design verification before fabrication. A single point of failure in any of these structures would be sufficient to prevent light propagation through the structure. Infrared imaging is a useful experimental tool to study light propagation through the length of these structures [34], [35]. Within the timeline of this project, a one-year supplementary collaboration with IBM's silicon photonics group supported by NSF (1028553) provided insights into silicon photonics fabrication and also supported technical collaboration on fabricating these structures. Led by graduate students supported on this project, several jointly-authored publications resulted from this collaboration [12], [36], [37].

Dr. Ivan B. Divliansky (Research Scientist, CREOL, Florida) collaborated with us for the electron-beam lithography of long waveguide structures. We sent chips spin-coated with resist to him for exposure using a Leica EBPG 5000+ ebeam system, and the chips were returned to us for developing and postprocessing. Jointly-authored publications resulted from this collaboration [34], [38], [39].

Novel measurement techniques which were very useful for studying CROWs were developed based on a single-scan, Jones matrix-based, interferometric spectral measurement instrument (Luna Tech. Optical Vector Analyzer OVA5000, generously loaned at no cost by Luna Technologies, VA and subsequently used in an innovative way in our measurements. A jointlyauthored publication resulted from this collaboration [12]. The high spectral resolution of measurements of transmission amplitude and group delay was useful in performing studies of optical transport across the passband [36].

Impact on Human Resources: Three students in the PI's group (one supported directly by this grant, one primarily supported by the PI's CAREER grant, and the third by an NSF Graduate Fellowship) have worked on this topic during the past year as part of their education and training. A post-doctoral scholar participated in this research and presented results at an invited talk at the SPIE Photonics West 2010 conference. Three undergraduates participated in summer research activities related to this project. One of the undergraduates was a Regents Scholar at UCSD and continued his research during the academic year. 


\section{Subsequent Extensions}

As part of this project, we studied and compared the single-resonator and coupled-resonator configurations from the viewpoint of quantum light generation [27]. The enhanced nonlinearity of the CROW structure was critical to our successful realization of entangled photon-pair and heralded singlephoton generation using silicon photonics [40]-[42]. However, subsequent research showed it was possible to monitor and control a single silicon microring resonator more precisely [43] compared to a long CROW device. FWM can also be performed efficiently using a single silicon microring if the nonlinear losses can be mitigated [44]. By progressively optimizing the design and the experiment, we eventually achieved a high quality of entangled photonpair generation using optimized single silicon microresonators, which outperformed the CROW device [45]. The CROW structure remains of interest, but by studying the $\chi^{(2)}$ nonlinearity in periodically-poled lithium niobate waveguides [46], an even higher level of performance as a pair-source (increased brightness and higher quality) was eventually achieved [47]. We showed that it is possible to include ferroelectric thin films of lithium niobate in the silicon photonics platform using bonding and layer transfer techniques [48]. Therefore, the impact of disorder is manifest differently. The research techniques and computational tools developed in this project for studying coupled-microring structures and understanding the effects of disorder continued to be useful in our research for many years after this project.

Figure 2 shows the progress achieved in making coupled-microring CROWs. In 2006, about 12 coupled polymer microrings were fabricated with a loss of about $2.5 \mathrm{~dB} /$ ring at $1.5 \mu \mathrm{m}$ wavelength [49]. IBM showed 28 rings with acceptable transmission in 2007, but transmission was severely degraded for longer CROWs [50]. This made the applications of CROWs in optical communications difficult to realize. This project achieved a significant improvement in the performance of coupled-microring CROWs, with a specific focus on disorder-tolerant designs for silicon microring CROWs. Structures up to 235 serially-coupled microrings were seen to successfully guide light. The loss per ring was as low as $0.06 \mathrm{~dB} /$ ring and a group delay of about $1 \mathrm{ps} /$ ring was obtained. A large ensemble of devices fabricated on two $200 \mathrm{~mm}$ SOI wafers was studied during this project.

\section{Open-Access Reporting Initiative}

PRAISE: This open-access document is provided in support of our PRAISE

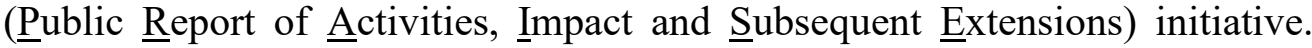
What is it? An open-access document shared with the public which describes 
the research outcomes of publicly-funded projects. For us, these projects are typically funded by the NSF (National Science Foundation).

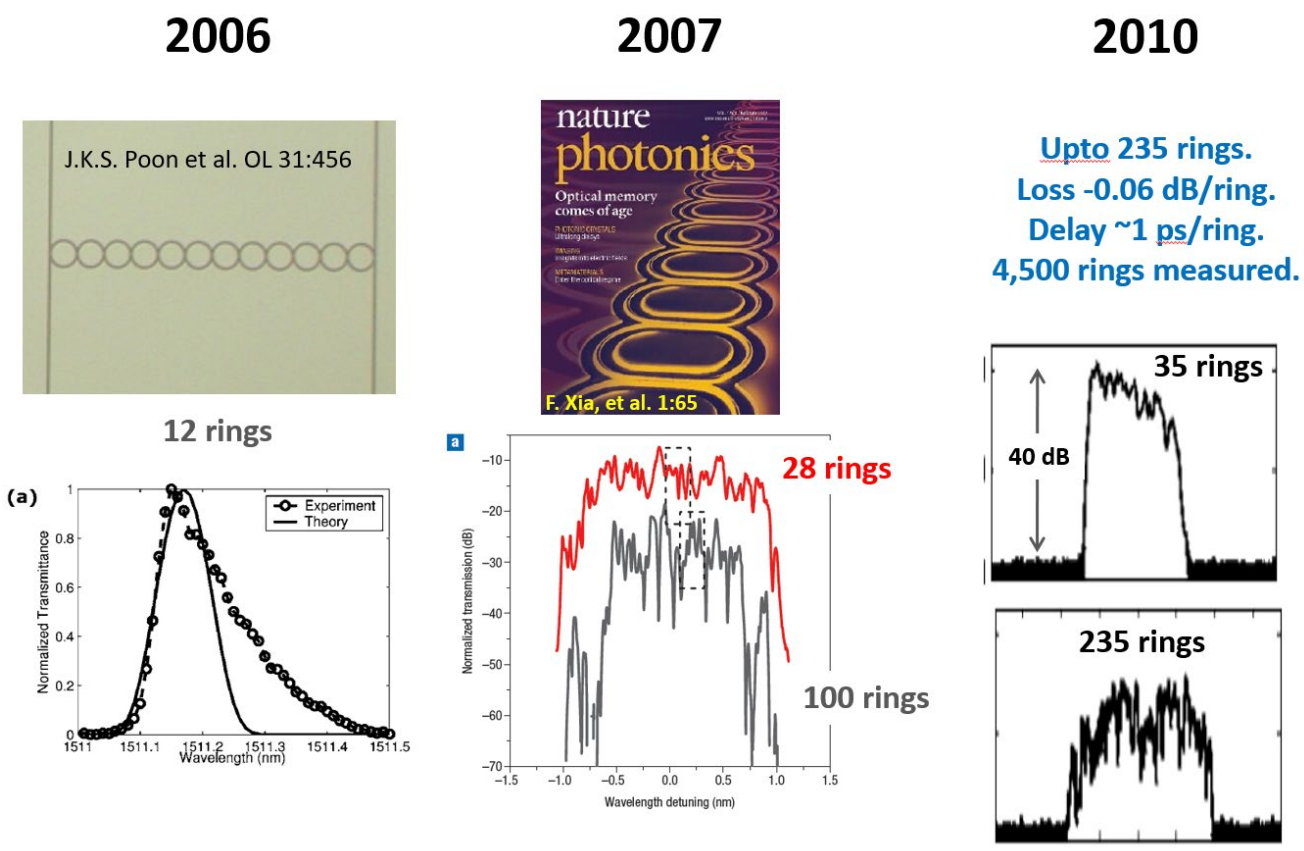

Figure 2 Progress in coupled-microring CROW structures between 2006-2010. The third column represents the accomplishments of this project.

\section{References}

[1] G. T. Reed, Silicon Photonics: The State of the Art. John Wiley \& Sons, 2008.

[2] J. M. Ziman, Models of disorder. Cambridge: Cambridge University Press, 1979.

[3] S. Mookherjea and A. Yariv, "Second-harmonic generation with pulses in a coupled-resonator optical waveguide," Phys. Rev. E, vol. 65, no. 2, p. 026607, Jan. 2002, doi: 10.1103/PhysRevE.65.026607.

[4] S. Mookherjea and A. Yariv, "Kerr-stabilized super-resonant modes in coupled-resonator optical waveguides," Phys. Rev. E, vol. 66, no. 4, p. 046610, Oct. 2002, doi: 10.1103/PhysRevE.66.046610.

[5] S. Mookherjea and A. Yariv, "Optical pulse propagation and holographic storage in a coupled-resonator optical waveguide," Phys. Rev. E, vol. 64, no. 6, p. 066602, Nov. 2001, doi: 10.1103/PhysRevE.64.066602.

[6] S. Mookherjea, "Semiconductor coupled-resonator optical waveguide laser," Appl. Phys. Lett., vol. 84, no. 17, pp. 3265-3267, Apr. 2004, doi: 10.1063/1.1719278. 
[7] R. Kumar, M. Savanier, J. R. Ong, and S. Mookherjea, "Entanglement measurement of a coupled silicon microring photon pair source," Opt. Express, vol. 23, no. 15, p. 19318, Jul. 2015, doi: 10.1364/OE.23.019318.

[8] M. L. Cooper et al., "235-ring Coupled-Resonator Optical Waveguides," in Conference on Lasers and Electro-Optics 2010, San Jose, California, 2010, p. CTuHH3. doi: 10.1364/CLEO.2010.CTuHH3.

[9] S. Mookherjea and M. A. Schneider, "Avoiding bandwidth collapse in long chains of coupled optical microresonators," Opt. Lett., vol. 36, no. 23, p. 4557, Dec. 2011, doi: 10.1364/OL.36.004557.

[10] M. L. Cooper and S. Mookherjea, "Modeling of Multiband Transmission in Long Silicon Coupled-Resonator Optical Waveguides," IEEE Photon. Technol. Lett., vol. 23, no. 13, pp. 872-874, Jul. 2011, doi: 10.1109/LPT.2011.2141657.

[11] S. Mookherjea and A. Yariv, "Pulse propagation in a coupled resonator optical waveguide to all orders of dispersion," Phys. Rev. E, vol. 65, no. 5, p. 056601, Apr. 2002, doi: 10.1103/PhysRevE.65.056601.

[12] M. L. Cooper et al., "Waveguide dispersion effects in silicon-oninsulator coupled-resonator optical waveguides," Opt. Lett., vol. 35, no. 18, p. 3030, Sep. 2010, doi: 10.1364/OL.35.003030.

[13] S. Mookherjea, "Dispersion characteristics of coupled-resonator optical waveguides," Opt. Lett., vol. 30, no. 18, p. 2406, Sep. 2005, doi: 10.1364/OL.30.002406.

[14] S. Mookherjea, "Using gain to tune the dispersion relation of coupledresonator optical waveguides," IEEE Photon. Technol. Lett., vol. 18, no. 5, pp. 715-717, Mar. 2006, doi: 10.1109/LPT.2006.871144.

[15] S. Olivier et al., "Miniband transmission in a photonic crystal coupledresonator optical waveguide," Opt. Lett., vol. 26, no. 13, pp. 1019-1021, 2001.

[16] W. J. Kim, W. Kuang, and J. D. O'Brien, "Dispersion characteristics of photonic crystal coupled resonator optical waveguides," Opt. Express, vol. 11, pp. 3431-3437, 2003.

[17] S. Mookherjea and A. Yariv, "Optical pulse propagation in the tightbinding approximation," Opt. Express, vol. 9, no. 2, p. 91, Jul. 2001, doi: 10.1364/OE.9.000091.

[18] M. Bayindir, B. Temelkuran, and E. Ozbay, "Tight-binding description of the coupled defect modes in three-dimensional photonic crystals," Phys. Rev. Lett., vol. 84, no. 10, pp. 2140-2143, 2000.

[19] T. Mukaiyama, K. Takeda, H. Miyazaki, Y. Jimba, and M. KuwataGonokami, "Tight-binding photonic molecule modes of resonant bispheres," Phys. Rev. Lett., vol. 82, no. 23, pp. 4623-4626, 1999.

[20] S. Mookherjea, D. S. Cohen, and A. Yariv, "Nonlinear dispersion in a coupled-resonator optical waveguide," Opt. Lett., vol. 27, no. 11, p. 933, Jun. 2002, doi: 10.1364/OL.27.000933. 
[21] S. Mookherjea, J. S. Park, S.-H. Yang, and P. R. Bandaru, "Localization in silicon nanophotonic slow-light waveguides," Nature Photon, vol. 2, no. 2, pp. 90-93, Feb. 2008, doi: 10.1038/nphoton.2007.278.

[22] Y. Lahini et al., "Anderson localization and nonlinearity in onedimensional disordered photonic lattices," Phys. Rev. Lett., vol. 100, p. 013906, 2008.

[23] R. Aguinaldo, Yiran Shen, and S. Mookherjea, "Large Dispersion of Silicon Directional Couplers Obtained via Wideband Microring Parametric Characterization," IEEE Photon. Technol. Lett., vol. 24, no. 14, pp. 1242-1244, Jul. 2012, doi: 10.1109/LPT.2012.2198639.

[24] J. R. Ong, R. Kumar, and S. Mookherjea, "Silicon microring-based wavelength converter with integrated pump and signal suppression," Opt. Lett., vol. 39, no. 15, p. 4439, Aug. 2014, doi: 10.1364/OL.39.004439.

[25] M. L. Cooper and S. Mookherjea, "Numerically-assisted coupledmode theory for silicon waveguide couplers and arrayed waveguides," Opt. Express, vol. 17, no. 3, p. 1583, Feb. 2009, doi: 10.1364/OE.17.001583.

[26] S. Mookherjea, "Spectral characteristics of coupled resonators," $J$. Opt. Soc. Am. B, vol. 23, no. 6, p. 1137, Jun. 2006, doi: 10.1364/JOSAB.23.001137.

[27] J. R. Ong and S. Mookherjea, "Quantum light generation on a silicon chip using waveguides and resonators," Opt. Express, vol. 21, no. 4, p. 5171, Feb. 2013, doi: 10.1364/OE.21.005171.

[28] A. Ciattoni, B. Crosignani, S. Mookherjea, and A. Yariv, "Nonparaxial dark solitons in optical Kerr media," Opt. Lett., vol. 30, no. 5, p. 516, Mar. 2005, doi: 10.1364/OL.30.000516.

[29] B. Crosignani, A. Yariv, and S. Mookherjea, "Nonparaxial spatial solitons and propagation-invariant pattern solutions in optical Kerr media," Opt. Lett., vol. 29, no. 11, p. 1254, Jun. 2004, doi: 10.1364/OL.29.001254.

[30] A. A. Sukhorukov and Y. S. Kivshar, "Spatial optical solitons in nonlinear photonic crystals," Physical Review E, vol. 65, p. 36609, 2002.

[31] S. Mookherjea and M. A. Schneider, "The nonlinear microring adddrop filter," Opt. Express, vol. 16, no. 19, p. 15130, Sep. 2008, doi: 10.1364/OE.16.015130.

[32] R. Aguinaldo et al., "Wideband silicon-photonic thermo-optic switch in a wavelength-division multiplexed ring network," Opt. Express, vol. 22, no. 7, p. 8205, Apr. 2014, doi: 10.1364/OE.22.008205.

[33] S. Mookherjea, J. R. Ong, X. Luo, and L. Guo-Qiang, "Electronic control of optical Anderson localization modes," Nature Nanotech, vol. 9, no. 5, pp. 365-371, May 2014, doi: 10.1038/nnano.2014.53. 
[34] M. L. Cooper, G. Gupta, J. S. Park, M. A. Schneider, I. B. Divliansky, and S. Mookherjea, "Quantitative infrared imaging of silicon-on-insulator microring resonators," Opt. Lett., vol. 35, no. 5, p. 784, Mar. 2010, doi: 10.1364/OL.35.000784.

[35] S. Mookherjea and H. R. Grant, "High dynamic range microscope infrared imaging of silicon nanophotonic devices," Opt. Lett., vol. 37, no. 22, p. 4705, Nov. 2012, doi: 10.1364/OL.37.004705.

[36] M. L. Cooper et al., "Statistics of light transport in 235-ring silicon coupled-resonator optical waveguides," Opt. Express, vol. 18, no. 25, p. 26505, Dec. 2010, doi: 10.1364/OE.18.026505.

[37] J. R. Ong et al., "Low-power continuous-wave four-wave mixing in silicon coupled-resonator optical waveguides," Opt. Lett., vol. 36, no. 15, pp. 2964-2966, 2011.

[38] Y. Shen, I. B. Divliansky, D. N. Basov, and S. Mookherjea, "Electricfield-driven nano-oxidation trimming of silicon microrings and interferometers," Opt. Lett., vol. 36, no. 14, p. 2668, Jul. 2011, doi: 10.1364/OL.36.002668.

[39] Y. Shen, I. B. Divliansky, D. N. Basov, and S. Mookherjea, "Perfect set-and-forget alignment of silicon photonic resonators and interferometers," in Optical Fiber Communication Conference/National Fiber Optic Engineers Conference 2011, Los Angeles, California, 2011, p. PDPC3. doi: 10.1364/OFC.2011.PDPC3.

[40] M. Davanço et al., "Telecommunications-band heralded single photons from a silicon nanophotonic chip," Appl. Phys. Lett., vol. 100, no. 26, p. 261104, Jun. 2012, doi: 10.1063/1.4711253.

[41] R. Kumar, J. R. Ong, M. Savanier, and S. Mookherjea, "Controlling the spectrum of photons generated on a silicon nanophotonic chip," Nat Commun, vol. 5, no. 1, p. 5489, Dec. 2014, doi: 10.1038/ncomms6489.

[42] R. Kumar, J. R. Ong, J. Recchio, K. Srinivasan, and S. Mookherjea, "Spectrally multiplexed and tunable-wavelength photon pairs at $155 \mu \mathrm{m}$ from a silicon coupled-resonator optical waveguide," Opt. Lett., vol. 38, no. 16, p. 2969, Aug. 2013, doi: 10.1364/OL.38.002969.

[43] M. Savanier, R. Kumar, and S. Mookherjea, "Optimizing photon-pair generation electronically using a $p-i-n$ diode incorporated in a silicon microring resonator," Appl. Phys. Lett., vol. 107, no. 13, p. 131101, Sep. 2015, doi: 10.1063/1.4932047.

[44] J. R. Ong, R. Kumar, R. Aguinaldo, and S. Mookherjea, "Efficient CW Four-Wave Mixing in Silicon-on-Insulator Micro-Rings With Active Carrier Removal," IEEE Photon. Technol. Lett., vol. 25, no. 17, pp. 1699 1702, Sep. 2013, doi: 10.1109/LPT.2013.2272521.

[45] C. Ma, X. Wang, V. Anant, A. D. Beyer, M. D. Shaw, and S. Mookherjea, "Silicon photonic entangled photon-pair and heralded single photon generation with CAR $>12,000$ and $\mathrm{g}^{\wedge}(2)(0)<0006$," Opt. 
Express, vol. 25, no. 26, p. 32995, Dec. 2017, doi:

10.1364/OE.25.032995.

[46] O. Alibart et al., "Quantum photonics at telecom wavelengths based on lithium niobate waveguides," Journal of Optics, vol. 18, no. 10, p. 104001, Sep. 2016, doi: 10.1088/2040-8978/18/10/104001.

[47] J. Zhao, C. Ma, M. Rüsing, and S. Mookherjea, "High Quality Entangled Photon Pair Generation in Periodically Poled Thin-Film Lithium Niobate Waveguides," Phys. Rev. Lett., vol. 124, no. 16, p. 163603, Apr. 2020, doi: 10.1103/PhysRevLett.124.163603.

[48] X. Wang, P. O. Weigel, J. Zhao, M. Ruesing, and S. Mookherjea, "Achieving beyond-100-GHz large-signal modulation bandwidth in hybrid silicon photonics Mach Zehnder modulators using thin film lithium niobate," APL Photonics, vol. 4, no. 9, p. 096101, Sep. 2019, doi: 10.1063/1.5115243.

[49] J. K. Poon, L. Zhu, G. A. DeRose, and A. Yariv, "Transmission and group delay of microring coupled-resonator optical waveguides," Opt. Lett., vol. 31, no. 4, p. 456, Feb. 2006, doi: 10.1364/OL.31.000456.

[50] F. Xia, L. Sekaric, and Y. Vlasov, "Ultracompact optical buffers on a silicon chip," Nature Photon, vol. 1, no. 1, pp. 65-71, Jan. 2007, doi: 10.1038/nphoton.2006.42. 